\title{
FOCALIZING SLOW NEUTRON BEAMS AT AND BELOW MICRON SCALES AND DISCUSSION ON BNCT (II)
}

\author{
Ignacio Molina de la Peña ${ }^{(1)}$, María L. Calvo ${ }^{(1)}$, Ramón F. Álvarez-Estrada ${ }^{(2)}$
}

1. Departamento de Óptica, Facultad de Ciencias Físicas, Universidad Complutense, 28040 Madrid, Spain.

2. Departamento de Fïsica Teórica, Facultad de Ciencias Físicas, Universidad Complutense, 28040

Madrid, Spain

Author Email Address

(1)imolinap@ucm.es

(2) mlcalvo@ucm.es

(3) rfa@ucm.es

\begin{abstract}
We study the propagation of slow neutrons and their waveguiding properties in certain suitable thin films and in polycapillary glass fibres, having in mind their possible use in Boron Neutron Capture Therapy (BNCT) of small tumours. In PBSI2017 (and subsequently, in Phosphorus, Sulfur and Silicon and the Related Elements Volume 193, Issue 2, pages 64-73 (2018), we presented a general overview and some specific proposals for improved focalization of slow neutrons (at and a bit below one micron, with propagation up to about $1 \mathrm{~m}$. with relatively small attenuation ), by omitting a good number of quantitative aspects. The present work, based upon our presentation in PBSI2018, extends our previous proposals in PBSI2017 and provides more quantitative descriptions of them. Neutron wavelengths versus characteristic dimensions of the waveguides in our proposals typically require quantummechanical analysis: specifically, the description of the confined neutrons by means of propagation modes. The confined propagation of incoming waves along waveguides, the evolution of the resulting propagation modes and related issues are investigated.Those features give rise to computational challenges, depending on the smallness of (neutron wavelengths/ characteristic dimensions). Approximate analytical formulae are provided for the corresponding quantum-mechanical probabilities. Numerical estimates and the results of some simulations are presented. Consequences of the latter for BNCT will be discussed briefly.
\end{abstract}

Keywords Short-scale neutron focusing/ possibilities for BNCT 


\section{NEUTRON OPTICS}

Slow neutrons are microscopic sub-atomic particles and, so, as they propagate in space, are described by wave functions and probability amplitudes and are subject to interference and diffraction phenomena, which constitutes the neutron optics field. If the neutron with energy $E$ moves in vacuum (or in air, as a first approximation), its de Broglie wavelength $\lambda_{d b}$ is given by:

$$
E=\frac{2 \pi^{2} \hbar^{2}}{m \lambda_{d b}{ }^{2}}
$$

Where $\hbar$ is Plank's constant and $m$ is the neutron mass. Let a slow neutron propagate through a material medium having (positive) density $\rho$ (nuclei $/ \mathrm{cm}^{3}$ ), of order $10^{22}\left(\right.$ nuclei $/ \mathrm{cm}^{3}$ ) for the materials of interest here (and about three orders of magnitude smaller, for air). We shall always suppose propagation to occur under conditions such that interference and diffraction become manifest and are not suppressed by incoherence effects $^{1}$. The wave function $\Psi(x, t)$ for a slow neutron at the position $x$ (used here, generically, to denote one, two or three spatial dimensions) at time $t$, as the former propagates through the medium, satisfies the Schrödinger equation:

$$
\begin{gathered}
{\left[\frac{-\hbar^{2}}{2 \mathrm{~m}} \nabla^{2}+\mathrm{V}(\mathrm{x})\right] \Psi(\mathrm{x}, \mathrm{t})=\mathrm{i} \hbar \frac{\partial \Psi(\mathrm{x}, \mathrm{t})}{\partial \mathrm{t}}} \\
\mathrm{V}(\mathrm{x})=\frac{2 \pi \hbar^{2}}{\mathrm{~m}} \mathrm{~b} \rho
\end{gathered}
$$

In Eq,(2) $\nabla^{2}$ is the Laplacian and $V(x)$ is the effective potential due to the nuclei in the medium on the slow neutron. Although, in principle, $V(x)$ depends on $x$, in practice it is allowed to regard the former as constant in each of the spatial regions of interest, in which the slow neutron is propagating (as the right-hand-side of Eq. (3) displays). In principle, disjoint spatial regions have different values for the product $b \rho$. In turn, $b$ is the (coherent) neutron-nucleus scattering amplitude at low energy in the corresponding spatial region. $b$ determines the interaction seen by the slow neutron as it propagates through the medium and it will be crucial for our studies here. 
For certain purposes, one can bypass the use of the Schrödinger equation by using the geometrical-optics approximation (by assuming suitable smooth oscillations of the neutron wave functions). In the latter, one introduces the neutron refractive index:

$$
\mathrm{n}^{2}=1-\frac{2 \pi \hbar^{2}}{\mathrm{~m}} \frac{\mathrm{b} \rho}{\mathrm{E}}
$$

In principle, $b$ has both a real and imaginary part (determined by the absorption of a slow neutron by the nuclei in the medium). To the extent that nuclear absorption be a small effect (to be taken into account duly at some stage, if required), and as a first approximation, we shall regard $b$ as real, in what follows. The order of magnitude of $b$ is about $10^{-13} \mathrm{~cm} . b$ is positive for the nuclei of almost all elements in the Periodic Table, except for a few: Although $b$ is negative for certain specific isotopes, we consider only the cases with negative $b$ for naturally occurring elements, like $\mathrm{H}, \mathrm{Li}, \mathrm{Ti}, \mathrm{V}, \mathrm{Mn}$ ("naturally occurring" meaning that the averaging over all isotopes of the corresponding element has been carried out). In Table 1, we display the value of $b$ for the main elements of interest here. $\mu$ is the linear coefficient, which determines how a flux of neutrons decays exponentially,as they propagate in a medium $\stackrel{21}{ }$.

\section{TABLE 1. MATERIALS AND RELATED NEUTRON PROPERTIES}

\begin{tabular}{|l|l|l|}
\hline Material & $b\left(\mathbf{1 0}^{-12} \mathbf{c m}\right)$ & $\mu\left(\mathrm{cm}^{-1}\right)$ \\
\hline $\mathrm{Ti}$ & $-0,344$ & 0,20 \\
\hline $\mathrm{Si}$ & 0,415 & 0,004 \\
\hline $\mathrm{P}$ & 0,513 & 0,004 \\
\hline $\mathrm{B}$ & 0,530 & 60 \\
\hline $\mathrm{O}$ & 0,580 & -- \\
\hline Air & -- & $5,7 \cdot 10^{-4}$ \\
\hline
\end{tabular}

For Boron, the value of $\mu$ and the imaginary part of $b$ are so large that the real part of $b$ can be regarded as negligible, as a first approximation. 
The order of magnitude of $V(x)$, for typical material media of interest here is of order $10^{-7}$ to $10^{-8} \mathrm{eV}$. For air, the value of $V(x)$ is about three orders of magnitude smaller.

\section{NEUTRON WAVEGUIDES: SOME GENERAL FEATURES}

The confined propagation of slow neutrons occurs mainly along certain crucial inner region (core) of the waveguide and it is described by means of certain specific solutions $\Psi(x, t)$ of the Schrödinger equation, named propagation modes. Specifically, a propagation mode $\Psi(x, t)=\varphi(x) e^{\frac{-i E t}{\hbar}}$ is a solution of Eq. (2) (with time dependence $e^{\frac{-i E t}{\hbar}}, E$ being the neutron energy), which oscillates and, so, describes a wave along the core. $\Psi(x, t)$ decays rapidly (say, exponentially) along the directions transverse to that in which confined propagation occurs, far from the core ( $X Y$ plane in the present model). The regions (in which that decay occurs) surrounding the core constitute the clad or cladding. Confined propagation of slow neutrons (with energy $E$ ) occurs mainly (but not exclusively) along the core (say, in the $Z$-direction). Those transverse directions reduce to one (say, the $X$ direction) for planar waveguides (two spatial dimensions, defined in $X Z$ plane in the present model). For waveguides with fibre-like structure they include both transverse directions ( $X$ and $Y$ ) (three spatial dimensions). Planar waveguides are formed by an inner region made up by a suitable material (the core, along the axis of the waveguide), surrounded by one or several regions (clad or cladding) composed by a different material with a smaller refractive index.

When confinement occurs, there is always one propagation mode (the so-called fundamental one). An important issue is whether other propagation modes exist, besides the fundamental one. Let $A$ be the total area of the core, in the $X Y$ plane. In general, the total number of propagation modes increases if $A \cdot \Delta(b \rho)$ increases. Notice that:

$$
A \cdot \Delta(b \rho)=\pi\left(\frac{A}{\lambda_{d b}{ }^{2}}\right)\left(\frac{V_{\text {clad }}-V_{\text {core }}}{E}\right)
$$

In the geometrical-optics approximation, confined propagation of slow neutrons occurs if the refractive index of the clad is smaller than that of the core: 


$$
\mathrm{n}_{\text {core }}>\mathrm{n}_{\text {clad }}
$$

An important ratio for thermal neutrons is $\left(\frac{V_{\text {clad }}-V_{\text {core }}}{E}\right)$, which is of the order of about $10^{-6}$. $A^{1 / 2}$ (which is of order of the transverse dimension of the core, (in the $X Y$ plane) has to be larger than $\lambda_{d b}$ by about a factor $10^{3}$.

Let us consider briefly a slow neutron approaching entrance face of a waveguide. Let $\theta$ be the angle formed by the neutron momentum with the axis $(Z)$ of the waveguide. There exists a certain characteristic angle $\overline{c r}$, named complement of the critical angle such that the neutron does propagate confined along the core if: $\theta<\overline{c r}$, .within the geometrical optics approximation. Then, total internal reflection of the neutron in the inner surface (core-cladding) occurs. For typical materials of interest here (like Ti...), $\theta_{c r}$ is of order $10^{-2}$ to $10^{-3}$ radians.,. Typically, the influence of the acceptance angle reduces the propagating flux $F_{\text {in }}$ by a factor of order $10^{-4}$, compared to $F_{0}$. Notice that this kind of reduction in the propagating flux, due to $\theta_{c r}$ value, is independent on (and has a different physical origin from) that arising from the beam attenuation represented by the linear coefficient $\mu$.

\section{OUR PREVIOUS PROPOSALS}

Previous experiments with $\mathrm{Ti}$ planar waveguides established slow neutron propagation experimentally in $1994^{15}$. The ratio of the thermal neutron flux detected after the waveguiding effect in the Ti layer over the measuring incoming flux, $I_{d} / I_{0}$, was about $3.5 \cdot 10^{-4}$, within the order of magnitude expected ${ }^{21}$._The confined neutron flux along $z$ was strongly attenuated beyond $20 \mathrm{~mm}^{21}$.

We made ${ }^{21}$ some proposals, based upon two coupled waveguides (TCW), for generating_slow neutron beams which, possibly, would propagate along longer distances (say, about $1 \mathrm{~m}$ ) in the $Z$-axis and be confined in transverse directions down to about a fraction of a micron. All proposals involve Ti as an adequate candidate, since: a) the values of $b$ and $\mu$ for it seem, in a global sense, more favorable than those for other possibilities (see Table 1), b) its natural abundance, c) pure samples of it can be prepared in a laboratory, d) its interesting mechanical, chemical and physical properties. 
It will be important to notice that, for air, the value of $b \rho$ is about three orders of magnitude smaller than for the typical elements used in cores and claddings.

The first proposal (TCW1) was based upon the successful experiment using a thin Ti film. The second proposal (TCW2), although not based upon experiments carried out previously, was a natural improving extension of TCW1 and of previous proposals in ${ }^{10-11}$. Both TCW1 and TCW2 could possibly have some application to BNCT, as we shall comment below.

\section{a. TCW1}

Two planar waveguides are successively coupled along the propagation direction Z.See Figure 1.

The first waveguide has a Ti film (core) and a clad formed by two films $\left(\mathrm{SiO}_{2}\right.$ and $\mathrm{Si}$ ). The whole device is placed in air: that is, there is air above $\mathrm{SiO}_{2}$ and below $\mathrm{Si}$. Confined propagation along $z$ occurs first in the Ti core, like in ${ }^{15}$. Transverse sizes along the $\mathrm{x}$ and $y$ directions could be similar to those in the experiment in Feng et al ${ }^{15}$. Anyway, we shall suppose that each of the $\mathrm{SiO}_{2}$ and $\mathrm{Si}$ films in the first waveguide have widths along $x$ larger than 0.5 microns: see the comment below regarding the widths of the $\mathrm{SiO}_{2}$ and $\mathrm{Si}$ films for the second waveguide. For simplicity in a first discussion, the clad above the $\mathrm{Ti}$ core reduces just to a $\mathrm{SiO}_{2}$ film. Notice that here, and contrary to the experiment in ${ }^{15}$, one is not interested in detecting neutrons leaving the Ti core and so there is one important difference regarding TCW1 compared with that of the experiment; its length along $Z$ is chosen to be definitely shorter than that one (say, less than $1 \mathrm{~cm})$, so as to reduce attenuation in the Ti core.

The second waveguide has air as core (with transverse dimensions similar to those of the first waveguide). The clad is similar to that of the first waveguide: two films $\left(\mathrm{SiO}_{2}\right.$ and $\left.\mathrm{Si}\right)$. The confinement in the core of the second waveguide follows from Eqs. (5-6), the very small value of $b \rho$ for air (core) and the larger positive value of $b \rho$ for the $\mathrm{SiO}_{2}$ and $\mathrm{Si}$ claddings (by about three orders of magnitude). However, the latter confinement is not strict, due to the finite widths of the $\mathrm{SiO}_{2}$ and $\mathrm{Si}$ claddings. In order that losses across the clad (by quantum mechanical tunneling across it towards air) be negligible, it is required that each of the $\mathrm{SiO}_{2}$ and $\mathrm{Si}$ films have widths along $x$ larger than 0.5 microns, as we shall now justify. Let any of the two films in the clad of the 
second waveguide have density $\rho$, coherent scattering amplitude $b>0$ and transverse width along $x$ equal to $d$. The probability for tunneling of a slow neutron from the air core of the second waveguide across a distance $d$ in one of its claddings is roughly proportional to $e^{-4(4 \pi b \rho)^{1 / 2} d}$, which is adequately small for $d>0.5$ microns.

The overall length of the second waveguide along $Z$ could be several tens of $\mathrm{cm}$ or even about $1 \mathrm{~m}$. Confined slow neutrons from the Ti core of the first waveguide would enter, possibly with very small attenuation, into the air core of the second waveguide. Since air has a small linear coefficient $\mu$ (see Table 1), a direct application of Eq. (1) in reference ${ }^{21}$ shows that there would be small attenuation in the core of the second waveguide and, hence, at its exit there would be an appreciable neutron flux.

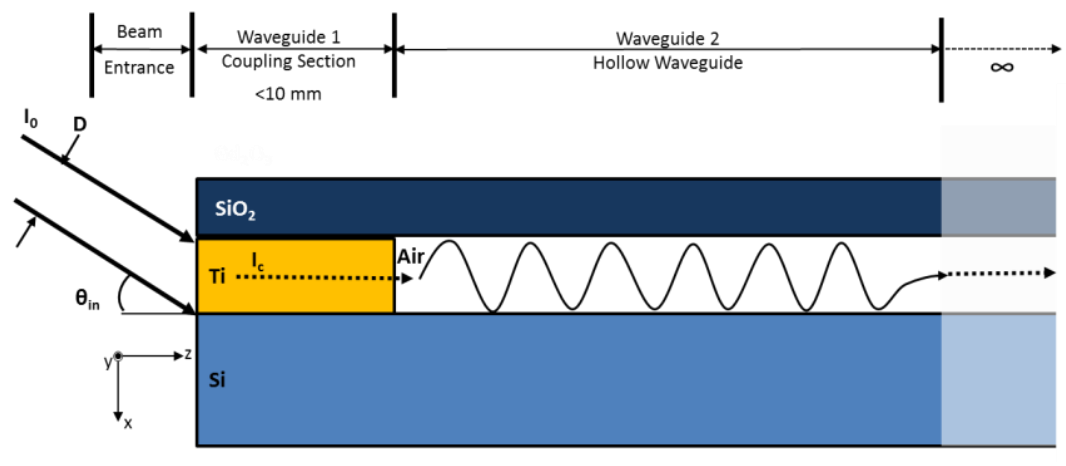

Figure 1. Schematic representation of the device in the TCW1 proposal.

At this stage, the structure of the first waveguide could be modified by illuminating laterally the Ti guide with the incoming neutron beam so as to become similar to that in the experiment in Feng et al ${ }^{15}$. This would facilitate the coupling of an incoming neutron to the propagation mode along the Ti core. See Figure 2.
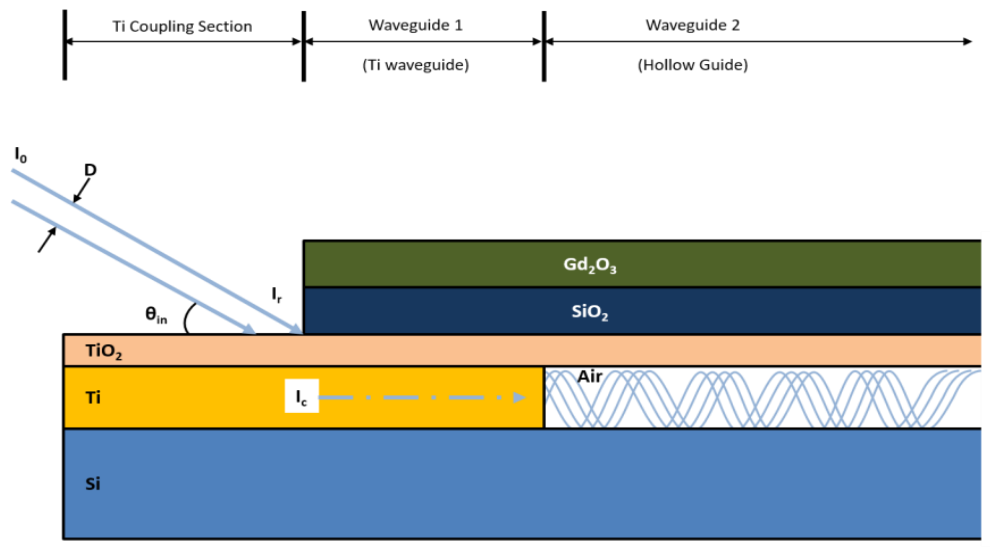

Figure 2. Schematic representation of the modified TCW1 proposal, with lateral neutron illumination improvement. 


\section{b. TCW2}

This proposed device also contains two coupled waveguides (see Figure 3) and which is placed in air. The first waveguide would be a single solid cylinder (with circular cross section), with $\mathrm{Ti}$ as inner core and $\mathrm{SiO}_{2}$ as surrounding clad. The diameter of the Ti core would be of the order of one micron and the width of the $\mathrm{SiO}_{2}$ clad would be of the same order. The length of the first waveguide should be smaller than $1 \mathrm{~cm}$. The second waveguide would be a hollow cylinder (with circular cross section), having air as inner core and $\mathrm{SiO}_{2}$ as surrounding clad as well. The width of the clad of the second waveguide could be similar to that of the first waveguide. In the coupling, the axis $Z$ in the $\mathrm{Ti}$ core of the first waveguide should coincide with the axis of the second waveguide. The length of the second waveguide could be some tens of $\mathrm{cm}$ and, possibly, about $1 \mathrm{~m}$. Neutrons, propagating confined along the Ti core of the first waveguide would enter into the air core of the second waveguide and propagate along the latter without appreciable attenuation along distances similar to those in the proposal TCW1, as another use of Eq. (1) in reference ${ }^{21}$ shows. The (strict) confinement in the core of the first waveguide follows from Eqs. (5-6) and the values of $b$ in Table 1. The confinement in the core of the second waveguide follows from Eqs. (5-6), the very small value of $b \rho$ for air (core) and the larger positive value of $b \rho$ for the $\mathrm{SiO}_{2}$ clad (by about three orders of magnitude). However, the latter confinement is not strict, due to the finite width of the $\mathrm{SiO}_{2}$ clad. Losses in the propagating neutron flux across the surrounding $\mathrm{SiO}_{2}$ clad of the second waveguide (by quantum mechanical tunneling across it towards air) would be negligible, since its width is larger than 0.5 microns: the justification is similar to the one for the clad of the second waveguide in the proposal TCW1.

In the present proposal TCW2, there would be confinement along both transverse directions $X$ and $Y$.

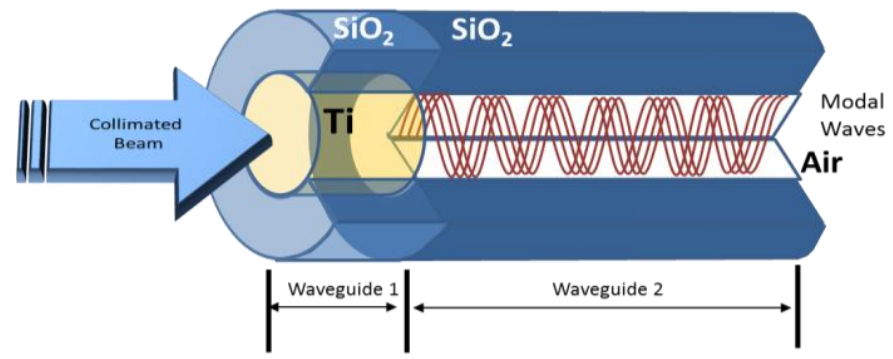

Figure 3. Schematic representation of the TCW2 proposed device. See text for details.

Received xx yyyy 2018; accepted xx yyyy 2018, Phosphorus Boron Silicon 2018 
We shall now be dealing with waveguides having transverse dimensions about one micron (and perhaps a fraction of it) and with thermal neutrons having smaller wavelengths (about some Angstroms), so that their quantum-mechanical nature has to be taken into account.

Several important and intriguing questions arise and should be understood regarding the wave-like behavior of the neutrons as they enter into and propagate as confined modes along the waveguide. In turn, the latter behavior influences the flux of confined neutrons which, subsequently, would leave the waveguide so as to be focused onto some small tumour, in a possible application to BNTC. Our work will be devoted towards providing information on such a flux which is the physical output. Accordingly, we shall study various aspects of how that confined propagation is generated and certain related issues.

\section{METHODS}

In our previous proposal ${ }^{21}$ we simulated the behaviour of a thermal neutron plane wave striking an aperture of $25 \AA$ and its diffraction pattern propagated on air with that for the propagation mode for a $\mathrm{Hg}(\mathrm{clad})$ - $\mathrm{Ti}$ (core, represented between black dotted lines) waveguide with similar arrangement (i.e., Ti guide aperture of $25 \AA$, surrounded by an $\mathrm{Hg}$ medium). Figure 4 displays the results of numerical simulations for a propagation mode.The figure represents the gain in the $\mathrm{Hg}-\mathrm{Ti}$ arrangement in comparison with air propagation in percentage, according to the formula:

$$
\text { Gain }=\frac{\varphi_{H g-T i}(x, z)-\varphi_{\text {air }}(x, z)}{\varphi_{\text {air }}(x, z)} \%
$$

Here, $\varphi(x, z)$ is the $t$-independent part of the corresponding propagation mode, in the actual thin film (in two spatial dimensions, $x$ and $z$ ): compare with section F. Both fields have been simulated by applying the Finite Difference Time Domain (FDTD) method to the Schrödinger equation. Notice that, just for the sake of the simulation, $\mathrm{Hg}$ has been used as the fiber clad (instead of $\mathrm{SiO}_{2}$ ) in order to maximize the confinement effect displayed, since $\mathrm{Hg}$ has the highest positive value of $b\left(b=1.27 \cdot 10^{-12} \mathrm{~cm}\right)$ in comparison with any other naturally occurring material. The wave attenuation effects 
due to incoherent scattering and nuclear absorption have not been introduced in the simulation in either of these two cases.

By inspecting Figure 4, one observes that there are no significant differences on the wave propagation along the first $1000 \AA$, since in this domain diffraction effects are dominant in both cases, because of the finite dimensions of the aperture. After some propagation, the intensity of the simulated field is reinforced inside the Ti guide and attenuated in the outer clad medium $(\mathrm{Hg})$, in comparison with what would be the case for neutron propagation in air. Propagating further, at $2000 \AA$ approximately, one sees that the propagation mode is formed inside the waveguide. Numerical simulations reveal that this is a slight effect, with a gain (achievement of confinement) for approximately $2 \%$ of the neutrons, which is physically intuitive since matter exhibits low interaction effects with neutrons (it is mostly transparent regarding their propagation). Nevertheless, even if $2 \%$ can be regarded as an acceptable (or high) gain, it will decrease significantly when attenuation effects (neutron capture, incoherent scattering, etc.) are introduced in the simulations equations. The counterpart of the $2 \%$ rise seen inside the Ti region is a 1-2\% lowering observed in the outer $(\mathrm{Hg})$ region, representing the loss of neutrons that have been confined in the guide.

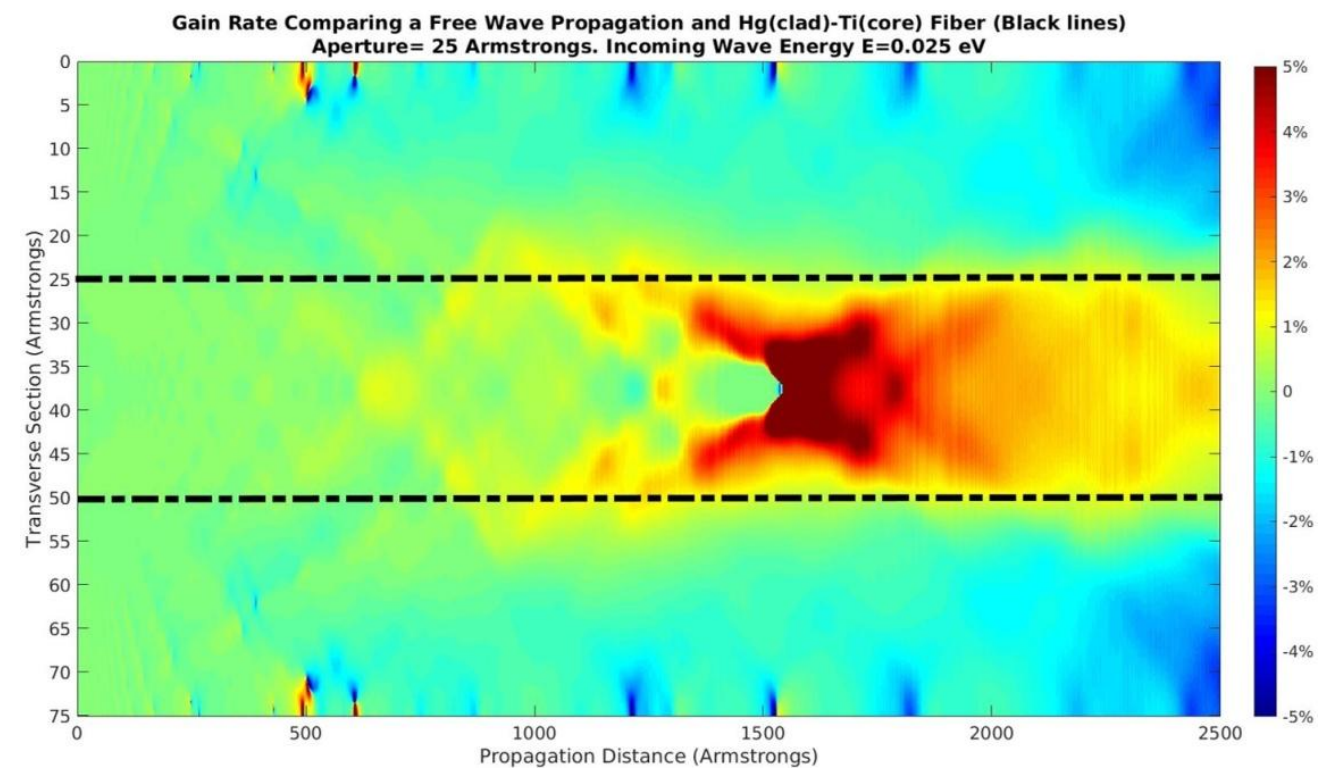

Figure 4. FDTD numerical simulation comparing the diffraction of a free neutron wave in a $25 \AA$ aperture with that for a propagation mode in a $25 \AA$ aperture $\mathrm{Hg}$-Ti waveguide. Ti core is represented between black dotted lines. 
This computation, by means of the direct use of FDTD, resulted to be computationally challenging, due to both computation time and memory consumption. For achieving these results, it cost around eighty hours of computation in a personal computer and involved matrices of $1 \mathrm{~Gb}$ average memory, which has resulted in finding the limitations of this method. If someone would try to apply it to larger scales (ie, a wider transverse section of the guide or longer propagation distances) the computing cost will be extremely high. Notice that the guides of our interest have an aperture of about $1000 \AA$ ad thus they require longer propagations distances for being well characterized.

The quantum-mechanical description of the confined neutrons by means of propagation modes can be approached, by making use of certain analogies with theoretical formulations in classical optics and associated results. The smallness of the ratio neutron wavelength versus characteristic dimensions of the fibers gives rise to computational challenges and different techniques must be used in order to estimate the effectiveness of the waveguide.

One simplified, but nevertheless interesting, case is that in which the neutron would suffer an infinite repulsive potential in the clad. Then, the wavefunction of the thermal neutron vanishes in the whole clad. The qualitative physical picture is that the incoming neutron wave, as it enters into one endface of a waveguide and starts to propagate, becomes increasingly extinguished while, if certain conditions are met, confined propagation modes are generated along the waveguide (without entering into the clad, which is excluded). Even this simplified case and its physical picture are rather difficult to formulate and solve quantitatively, in a compact way. The shall outline some essentials of a new formulation (omitting details and justifications) and present and discuss the results of several simulations.

These issues have led us to the analysis of an ideal case (infinite repulsive potential in the clad) by means of the use of Green's Theorem as in Eq.(9) in order to develop a new simulation procedure that let us improve the simulation cost.

\section{a. CLAD WITH INFINITELY REPULSIVE POTENTIAL}


We start with the scheme represented in Figure 5 in which we assume a semiinfinite two-dimensional waveguide in the $Z X$ plane, having aperture $x_{0}$ along the $X$ axis, centered at the origin of coordinates and extending along $Z-$ axis and parallel to it, from $z=0$ to $z=+\infty$. Ideally, the potential inside and along this guide is assumed to be $V=0$ (i.e., no material), as well as in the zone of wave incidence $z<0$. The clad of this guide is assumed to be an ideal repulsive potential $V=+\infty$, in $z \geq 0, x \geq$ $\pm x_{0} / 2$. This, in turn, is formulated by imposing Dirichlet boundary conditions $(\varphi=0)$ in the four guide walls $\left(z=0, x \geq+x_{0} / 2\right),\left(z=0, x \leq-x_{0} / 2\right)$ and $(z \geq 0, x=$ $\pm x_{0} / 2$ ), We assume an incoming plane wave in normal direction to the aperture from $z=-\infty$ in the form of $\varphi(x)=e^{i K_{z} z}$ with a well defined wavevector $K_{z}>0$.

Our aim is to find the wave function that solves the partial differential equation for $\varphi(z, x)$ following from (3), with Dirichlet boundary conditions and corresponding to the above incoming plane wave. There is no known method yielding in compact form the solution $\varphi(z, x)$ of this problem. However, we have been able to recast the determination of $\varphi(z, x)$ in terms of a suitable inhomogeneous linear integral equations containing the former, certain functions $\mu_{i}(x)$, the incoming thermal neutron wavefunction and certain Green function given below $(G)$.

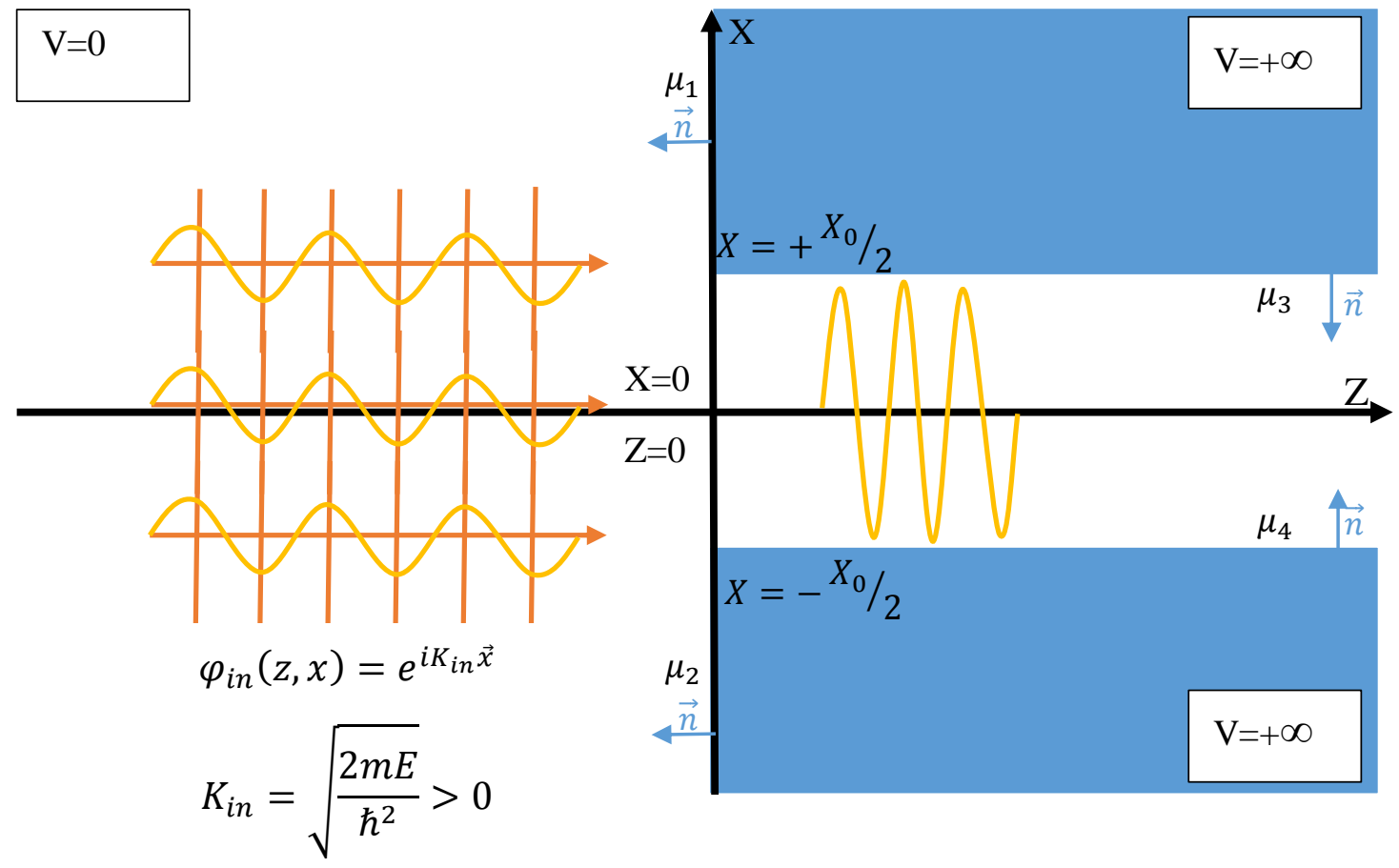


Figure 5. Scheme of the semiinfinite ideal waveguide with infinitely repulsive potential in the clad, showing the main parameters associated to the neutron confinement description.

Thus, we propose $\varphi(z, x)$ to be represented as :

$$
\begin{gathered}
\varphi(z, x)= \\
\varphi_{\text {in }}(z, x)-\left.\int_{x_{0} / 2}^{\infty} d x^{\prime} \frac{\partial G\left(z-z^{\prime}, x-x^{\prime}\right)}{\partial z^{\prime}}\right|_{z^{\prime}=0} \mu_{1}\left(x^{\prime}\right)-\left.\int_{-\infty}^{-x_{0} / 2} d x^{\prime} \frac{\partial G\left(z-z^{\prime}, x-x^{\prime}\right)}{\partial z^{\prime}}\right|_{z^{\prime}=0} \mu_{2}\left(x^{\prime}\right)- \\
\left.\int_{0}^{\infty} \mathrm{dz}^{\prime} \frac{\partial \mathrm{G}\left(\mathrm{z}-\mathrm{z}^{\prime}, \mathrm{x}-\mathrm{x}^{\prime}\right)}{\partial \mathrm{x}^{\prime}}\right|_{\mathrm{x}^{\prime}=\left(\mathrm{x}_{0} / 2\right)} \mu_{3}\left(\mathrm{z}^{\prime}\right)+\left.\int_{0}^{\infty} d z^{\prime} \frac{\partial G\left(z-z^{\prime}, x-x^{\prime}\right)}{\partial x^{\prime}}\right|_{x^{\prime}=\left(-x_{0} / 2\right)} \mu_{4}\left(z^{\prime}\right)
\end{gathered}
$$

Where $G\left(z-z^{\prime}, x-x^{\prime}\right)$ is the Green function associated to propagation in two dimensions:

$$
\mathrm{G}\left(\mathrm{z}-\mathrm{z}^{\prime}, \mathrm{x}-\mathrm{x}^{\prime}\right)=-\int \frac{\mathrm{d} \mathrm{K}_{\mathrm{z}}^{\prime} \mathrm{d} K_{\mathrm{x}}^{\prime}}{(2 \pi)^{2}} \frac{\exp \mathrm{i}\left[\mathrm{K}_{\mathrm{z}}^{\prime}\left(\mathrm{z}-\mathrm{z}^{\prime}\right)+\mathrm{K}_{\mathrm{x}}^{\prime}\left(\mathrm{x}-\mathrm{x}^{\prime}\right)\right]}{\mathrm{E}+\mathrm{i} \varepsilon-\frac{\hbar^{2}}{2 \mathrm{~m}}\left(\mathrm{~K}_{\mathrm{z}}^{\prime 2}+\mathrm{K}_{\mathrm{x}}^{\prime 2}\right)}
$$

$\varphi_{i n}(z, x)=e^{i K_{z} z} e^{i K_{x} x}$ is the initial incoming wave striking the waveguide. $\mu_{1}, \mu_{2}, \mu_{3}, \mu_{4}$ are certain functions defined in the waveguide walls (see figure 1) in which we will impose Dirichlet boundary conditions in order to find those functions. $E=\frac{\hbar^{2} K_{i n}}{2 m}$ is the total energy of the incoming wave (this will not change since the problem here implies no potential value). Notice that we have already imposed the sign of every integral depending of the direction of the normal vector to the surface where $\mu_{i}$ is defined (see figure 5).

A system of equations for $\mu_{1}, \mu_{2}, \mu_{3}, \mu_{4}: \mu_{i}=f\left(\mu_{j}, \mu_{k}, \mu_{l}\right) ; i \neq j, k, l$, can be obtained. We omit these equations, since the purpose of this paper is centered about BNCT development.

This system of equations for $\mu_{1}, \mu_{2}, \mu_{3}, \mu_{4}$, is very useful and interesting for the following reasons: a) they can be solved by successive iterations (thereby yielding an infinite series solution for $\varphi(z, x))$, b) one can set up simulations in order to compute numerically the low order iterations, c) the successive iterations allow for physically appealing interpretations of the propagation of the neutron wave along the core. 
One simplification is to make a physical approach of the form of the functions $\mu_{i}$ and check whether the final result obeys or not the boundary conditions of the problem. By making such a simplifications (proposing plane waves and constant fucntions) one obtained a formula that relates the number of propagation modes that these guides would accept with its aperture and the incoming wave energy. Thus, for an incoming plane wave with a fixed energy $E=\frac{\hbar^{2}}{2 m} K_{i n}^{2}$ the highest order mode achievable is a function of the guide aperture:

$$
\mathrm{N}_{\max }=\frac{\mathrm{X}_{0}}{\pi \hbar^{2}} \sqrt{2 \mathrm{mE}}
$$

Figure 6 shows the results of the maximum number of modes allowed in these fibres as a function of the neutron incoming energy and the aperture of the guide:

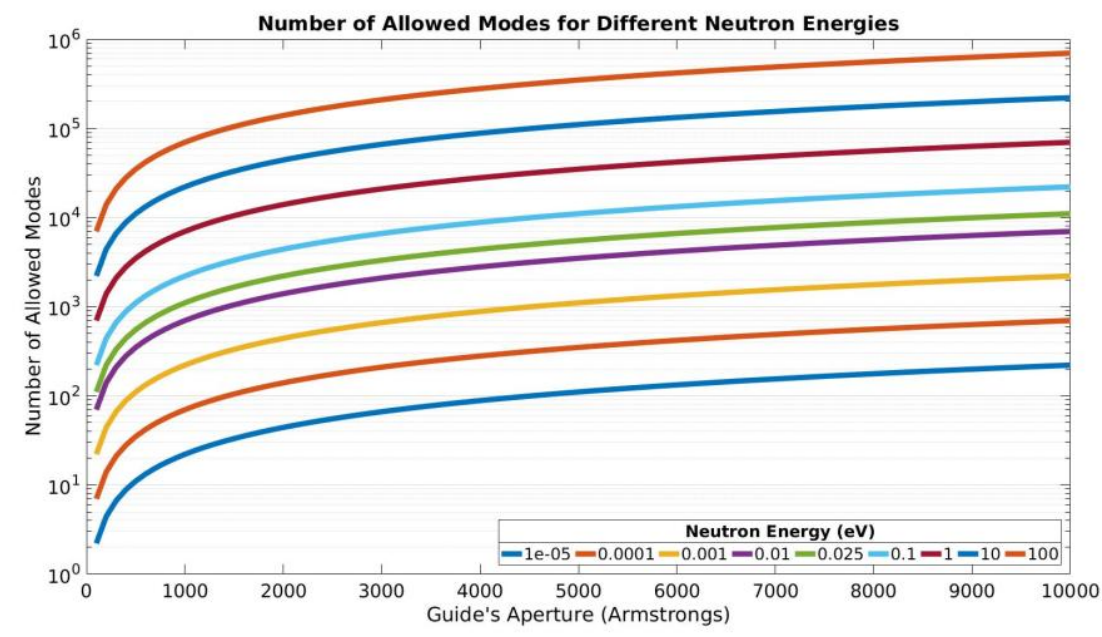

Figure 6. Number of allowed modes in waveguides having clad with infinite repulsive potential, depending on their aperture and incoming wave energy

The simulations for the system of equations for $\mu_{1}, \mu_{2}, \mu_{3}, \mu_{4}$ become much less costly in terms of computation than the direct use of FDTD procedure early mentioned since, unless it be necessary to perform a large number of terations, one only has to take into account the values and matrices generated at the wall of the waveguide (ie, one only has to solve each $\mu_{i}$ in order to characterize completely the wavefunction inside the guide). 
Figure 7 shows the results of a simulation for a neutron beam with an energy of $0.025 \mathrm{eV}$ entering in a waveguide with aperture $100 \mathrm{~nm}(1000 \AA)$ and after performing four iterations. One can see that there is a vanishing of the wavefunction at the entrance of the waveguide, meaning that the neutron probability is lost (i.e., the formation of propagation modes for a neutron is not achieved). Beyond that, one can observe some filaments reflecting that, due to interaction with the walls, propagation modes are achieved.

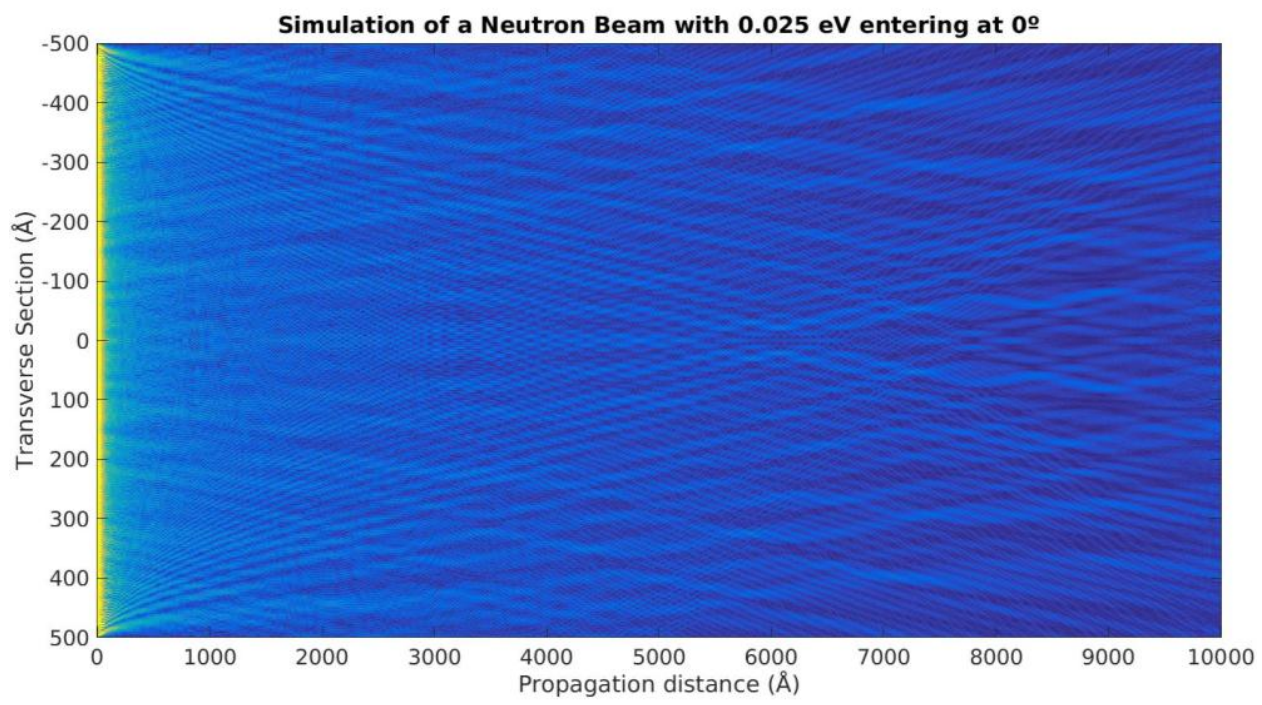

Figure 7. Numerical simulation of a neutron flux with energy $0.025 \mathrm{eV}$ impinging at $0^{\circ}$ propagation in a waveguide of infinitely repulsive potential clad 
In Figure 8, we show qualitatively the behaviour of the algorithm. In this case, the same neutron beam with energy $0.025 \mathrm{eV}$ impinging at $30^{\circ}$ in the entrance of the waveguide is simulated in sucessive iterations of the algorithm. One can observe that each iteration updates the information with the reflection produced in the wall by the beam. Notice that it is very similar to the behaviour expected in classical optics, but it is not the same since, in the present ranges of distances, diffraction effects are observable.
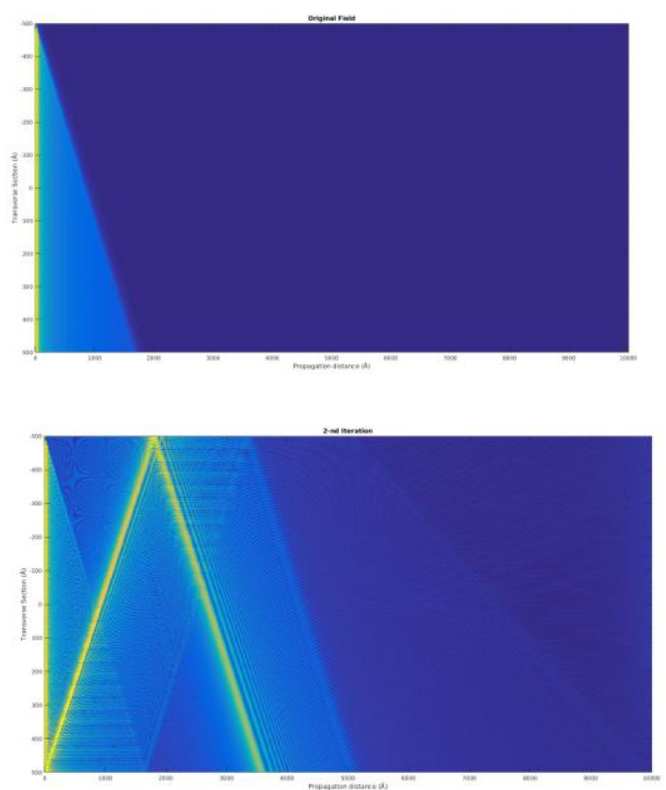
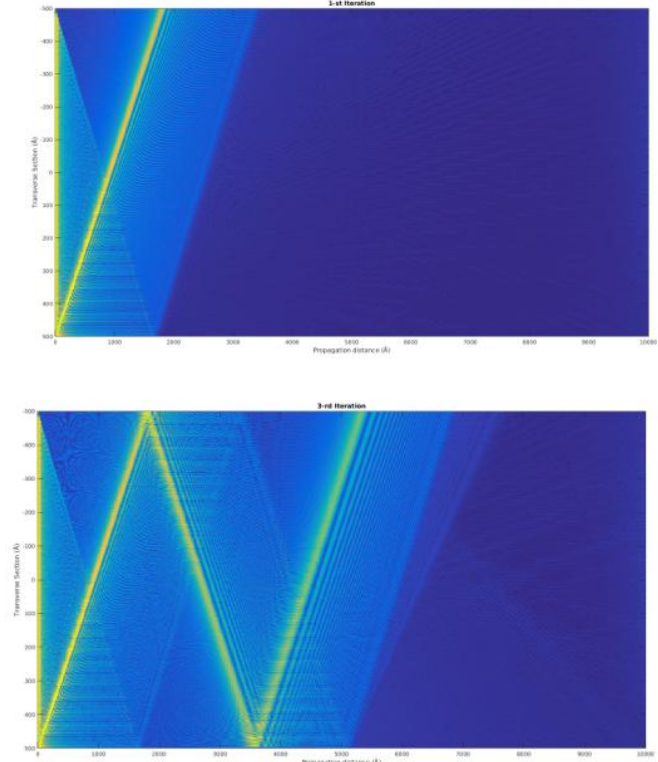

Figure 8. Numerical simulation of a neutron flux with energy $0.025 \mathrm{eV}$ impinging at $30^{\circ}$. It is shown the sequence of one, two, three and four iterations, respectively (from up left to right down).

Notice that in both cases the sizes involved in the present simulations for the waveguide are $1000 \AA$ of aperture and $10.000 \AA$ of propagation length. This, compared to the results obtained in the previous paper, constitutes an improvement of three orders of magnitude in the size of the present simulations, as achieved on a personal computer. Appart from that, each of these simulations took about 20 minutes of computing time, that represents a huge improvement compared to eighty hours needed for the simulations displayed in Figure 4.

\section{b. CLAD WITH FINITE REPULSIVE POTENTIAL}

The numerical estimation of a realistic confinement case, in which material has a low interaction with neutrons instead of the use of infinite potential, needs a deeper study. One can make some good asumptions obtaining approximate but accurate 
behaviour applying the algorithm exposed by Snyder and Love ${ }^{22}$. This algorithm has proved to be effective in the case of weakly-coupled waveguides that are our case (take into account that while the thermal neutron energy is of the order of $10^{-3} \mathrm{eV}$ the potential of a material medium is of the order of $10^{-5} \mathrm{eV}$ ). This algorithm is made by two steps:

- Step 1: Consider the wave from the medium 1 impinging the medium 2 assumed to be made of the material of the core of the waveguide. In this case, the classical refraction law is used to describe the wave in the medium 2 using Fresnel coefficients.

- Step 2: Once computed the refracted wave, you take into account the clad and fit the projection of the wave at medium 2 on the normalized propagation modes for a infinite waveguide.

Our analytical results for this algorithm (not derived here for brevity) is on Eq. (11):

$\int \mathrm{dx} \phi_{2}{\widehat{\phi_{2, n}}}^{*}=\left\{\begin{array}{l}A\left[\frac{\sin \left(\left(K_{x}+\chi_{n}^{\prime}\right) \frac{x_{0}}{2}\right)}{K_{x}+\chi_{n}^{\prime}}+\frac{\sin \left(\left(K_{x}-\chi_{n}^{\prime}\right) \frac{x_{0}}{2}\right)}{K_{x}-\chi_{n}^{\prime}}\right] \text { (even modes) } \\ \frac{A}{i}\left[\frac{\sin \left(\left(K_{x}+\chi_{n}^{\prime}\right) \frac{x_{0}}{2}\right)}{K_{x}+\chi_{n}^{\prime}}-\frac{\sin \left(\left(K_{x}-\chi_{n}^{\prime}\right) \frac{x_{0}}{2}\right)}{K_{x}-\chi_{n}^{\prime}}\right] \text { (odd modes) }\end{array}\right.$

Where $A$ is the normalization factor for an ortonormal mode corresponding to the potential well problem (see ${ }^{24}$ ) with a $\chi_{n}^{\prime}$ transverse wavenumber $x_{0}$ is the guide aperture and $K_{x}=K_{i n} \sin \theta$ is the component of the incident wave perpendicular to the separation plane of both mediums.

The excitation of the different modes and their contribution to the total value of the flux is represented in figure 9, where is simulated a semiinfinite waveguide of aperture $1000 \AA$ with a core of titanium surrounded by a clad of silicon. 


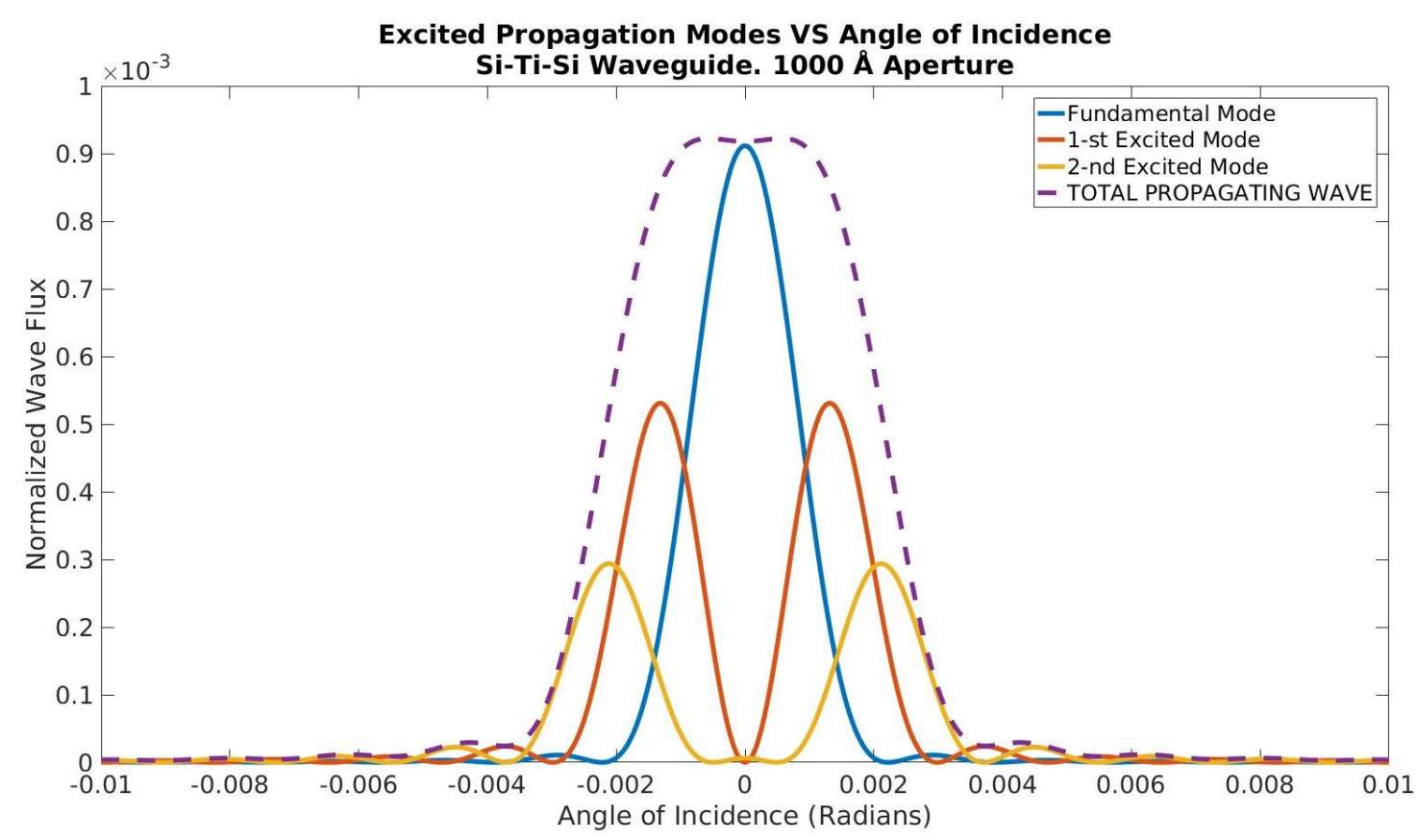

Figure 9. Numerical simulation of a neutron flux with energy $0.025 \mathrm{eV}$ impinging at different angles and the excited modes risen.

Inspecting the figure 9 one can appreciate the good correspondence with the classical behavior of an optical waveguide. Here the critical angle is estimated on $\theta_{c r}=3 \cdot 10^{-3}$ rad that implies a reduction of the flux by $10^{-3}$.

\section{c. TCW1, TCW2 and BNCT}

In our previous paper ${ }^{21}$ we mentioned that, BNCT (Boron Neutron Capture Therapy) is a therapeutic modality for treating certain malignant tumours, like primary brain tumours (glioblastomas) and head and neck cancer. In short, in order to start the treatment, certain tumour- localizing drug containing the (non-radioactive) isotope $B_{5}^{10}$ is injected into the patient in the form of BSH (sulfhydryl borane, with chemical formula $\mathrm{Na}_{2} \mathrm{~B}_{12} \mathrm{H}_{11} \mathrm{SH}$ ) and BPA (an amino acid: a dihydroxyboryl derivative of phenilalanine). At a subsequent stage, the patient is irradiated with a slow neutron beam (epithermal, thermal), focused towards the location of the malignant tumour, to which the above drug has been delivered previously. The basic BNCT (nuclear) reactions are:

$$
n+B_{5}^{10} \rightarrow H e_{2}^{4}+L i_{3}^{7} \text { and } n+B_{5}^{10} \rightarrow H e_{2}^{4}+L i_{3}^{7}+\gamma .
$$


2.8 $\mathrm{MeV}$ and $2.3 \mathrm{MeV}$ are respectively liberated in those two reactions, in the form of kinetic energies of the final $\mathrm{He}_{2}^{4}$ and $\mathrm{Li}_{3}^{7}$ particles that are able to produce damage to DNA macromolecule of a malignant cell.

A very important aspect is to control, as much as possible, the delivery of neutrons to malignant cells and, so, to avoid that normal tissue could receive the neutron radiation and it is the basic aim of this investigation. For a thermal neutron beam $F$ to be effective in standard BNCT, its flux $F$ should be not smaller than $5 \cdot 10^{8}$ (neutrons $\left./\left(\mathrm{cm}^{2} \cdot \mathrm{s}\right)\right)$.

Let us consider the accelerator-based neutron source, constructed by the private Japanese company Sumitomo and interesting for our discussion here, namely, the 30 $\mathrm{MeV} \mathrm{H}^{-}$Cyclotron ${ }^{21}$. It employs a proton beam equivalent to $1 \mathrm{~mA}$ and produces a flux $10^{9}$ (neutrons $/\left(\mathrm{cm}^{2} \cdot \mathrm{s}\right)$ ) (stable during about 30 minutes), which is specifically designed for BNCT.

Let the latter flux enter into the first waveguide in the above proposal TCW1 (see figure 2 and consider confinement along the transverse direction $X$ ), for definiteness. Then, plausibly, the slow neutron flux at the exit of the first waveguide, just at the entrance of the second waveguide, could be about $10^{5}$ (neutrons $/\left(\mathrm{cm}^{2} \cdot \mathrm{s}\right)$ ). If losses upon entering into the second waveguides are moderately small, as one would expect and as argued above, the outgoing flux at the exit of the second waveguide, having air core, should not suffer further appreciable attenuation, say, could be about $10^{4}\left(\right.$ neutrons $\left./\left(\mathrm{cm}^{2} \cdot \mathrm{s}\right)\right)$.

Let us now turn very briefly to TCW2 (see figure 3 and consider lateral confinement in both transverse directions $X$ and $Y$ ). A direct application of the $\theta_{c r}{ }^{2}$ reduction factor in the confined flux (subsection $\mathrm{F}$ in the preceding section) would suggest an outgoing flux at the exit of the second waveguide (having air core) comparable to the above one in the TCW1 proposal. One could also speculate that, also for TCW2, the coupling of the incoming neutrons to the propagation modes in the $\mathrm{Ti}$ core of the first waveguide could be enhanced adequately (perhaps, through some lateral illumination): a discussion of this aspect lies outside our scope here. Anyway, one could entertain the possibility that the outgoing flux at the exit of the second waveguide in TCW2 could be useful, possibly, for BNCT of small tumours, where smaller fluxes could suffice, as discussed above. 


\section{DISCUSSIONS AND CONCLUSIONS}

Our proposals are based upon earlier experiments by Feng et al. displaying slow neutrons confined propagation in thin films of short length (not exceeding $2 \mathrm{~cm}$ ) ${ }^{15}$ and extending previous theoretical proposals in three-dimensional fibre waveguides ${ }^{10-11}$. We present some proposals which could enable, possibly, confined propagation along distances about $1 \mathrm{~m}$. and their focalization at the micron scales and somewhat below. Our proposals, named TCW1 and TCW2, consider two suitably coupled waveguides. TCW1 is formulated in two dimensions and is based upon experiments with thin films: it describes confinement in $X$ direction and propagation in $Z$ direction. Moreover, TCW2 is formulated in three dimensions and is based upon previous proposals for thin fibres: it describes confinement in $X$ and $Y$ directions and propagation in $Z$ direction. In both TCW1 and TCW2, the first waveguide (with a length less than $1 \mathrm{~cm}$ ) has Ti in its core, in order to ensure proper confinement, and the second waveguide has air in its core, so as to ensure small attenuation. The claddings in both could well contain Si. For an incoming slow neutron flux about $10^{9}$ (neutrons $/\left(\mathrm{cm}^{2} \cdot \mathrm{s}\right)$ ) entering the first waveguide in either TCW1 or TCW2, our estimates would suggest that the outgoing one (at the exit of the second waveguide) could possibly be not smaller than about $10^{4}$ (neutrons $/\left(\mathrm{cm}^{2} \cdot \mathrm{s}\right)$ ). We entertain the possibility that the latter outgoing flux, even if small, could possibly be useful for BNCT of small tumours (with sizes of order about $1 \mathrm{~mm}$ or smaller).

To conclude, we would like to emphasize the, a priori, general interest of pursuing the exploration of shorter scales by means of slow neutrons and that, in the specific case of BNCT, improvements (whatever the device employed) in focalizing neutrons could enable to concentrate them onto malignant tissues and to reduce their delivery to normal tissues.

\section{ACKNOWLEDGEMENTS}

M. L. Calvo acknowledges Spanish Ministry of Economy and Competitiveness under Grant TEC2014-57394-P. R. F. Álvarez-Estrada acknowledges the financial support of Project FIS2015-65078_C2-1-P, Ministry of Economy and Competitiveness, Spain. We are grateful to Prof. Clara Viñas for having encouraged that our work (by 
virtue of the relationship of the proposals in it to BNCT) be communicated to PBSI2018

. We are also grateful to Mr. David Fernández for kind help.

\section{REFERENCES}

1. Byrne J.. Neutrons, Nuclei and Matter: An Exploration of the Physics of Slow Neutrons, IoP Publishing, Bristol 1995.

2. Sauerwein, W.; Moss, R.; Wittig, A. Research and Development in NCT, Monduzzi Editore, Bologna, 2002.

3. Barth, R.F. Appl. Radiat. Isot. 2009, 67, pp 53-56.

4. Hopewell, J. W.; Morris, G. M.; Schwint, A.; Coderre, J. A. Appl. Radiat. Isot. 2011, 69, pp 17561759.

5. Moss, R. L. Appl. Radiat. Isot. 2014, 88, pp 2-11.

6. Erko, A.; Idir, M.; Krist, T.; Michette, A. G., Modern Developments in X-Ray and Neutron Optics] (Eds. Erko, A.; Idir, M.; Krist, T.; Michette, A. G.); Springer: Berlin, 2010, pp 1-5.

7. Sauerwein W., Bet P.M., Wittig A. Neutron Capture Therapy. Principles and Applications (Eds. Sauerwein W., Wittig A., Moss R., Nakagawa Y), Springer: Berlin 2012. pp 117-161.

8. Ott, F. Modern Developents in X-Ray and Neutron Optics (Eds. Erko, A.; Idir, M.; Krist, T.; Michette, A. G.); Springer: Berlin, 2010, pp 113-135.

9. De Wames, R. E.; Sinha, S. K. Phys. Rev. B 1973, 7, pp 917-921.

10. Alvarez-Estrada, R. F.; Calvo, M. L J. Phys.D: Appl. Phys., 1984, 17, pp 475-502. Ibid: . J. Phys., 1984, 45 (Suppl.3), pp C3-243-C4-248.

11. Calvo, M. L.; Alvarez-Estrada, R. F J. Phys.D: Appl. Phys. , 1986, 19, pp 957-973.

12. Kumakhov, M. A.; Komarov, F. F. Phys. Rep. 1990, 191, pp 289-350.

13. Kumakhov, M. A.; Sharov, V. A. Nature 1992, 357, pp 390-391.

14. Chen, H.; Downing, R. G.; Mildner, D. F. R.; Gibson, W. M. ; Kumakhov, M. A. ; Ponomarev, I. Yu.; Gubarev, M. V. Nature. , 1992, 357, pp 391-393.

15. Feng, Y. P.; C. F. Marjkrzak, S. K. Sinha, D. G. Wiesler, H. Zhang and H. W. Deckman Phys. Rev. B 1994, 49(15), pp 10814-10817.

16. Pogossian, S. P.; Menelle, A. Le Gall, H. ; Desvignes, J. M.; Artinian, M. Phys. Rev. B 1996, 53, pp 14359-14363.

17. Menelle, A.; Pogossian, S. P.; Le Gall, H. ; Desvignes, J. M.; Ben Youssef, J. Physica B, 1997, $234-$ 236, pp 510-512.

18. Kozhenikov S. V.; Khaydukov, Yu. N.; Keller, T.; Ott, F.; Radu, F. arXiv: 1511.07286v2[condmat.mtr1-sci] 24 Nov. 2015

19. Alvarez-Estrada, R. F.; Calvo, M. L. Appl. Radiat. Isot. , 2004, 61, pp 841-844.

20. Alvarez-Estrada, R. F.; Calvo, M. L. Optical Waveguides. From Theory to Applied Technologies (Eds. Calvo, M. L. and Lakshminarayanan, V.), CRC Press: Boca Ratón, 2007, pp 331-385. 
21. Alvarez-Estrada, R. F.; Molina de la Peña, I.; Calvo, M. L. Phosphorus, Sulfur and Silicon and theRelated Elements 2018193 (Issue 2) pp 64-73.

22. Snyder, A. W.; Love, J.; Optical Waveguide Theory, Springer, 1983.

23. Mitsumoto, T.; Fujita, K.; Ogasawara, T.; Tsutsui; H.; Yajima, S.; Maruhashi, Sakurai, Y.; Tanaka, H.; Proc. of Cyclotrons and their Applications (International Conference CYCLOTRON 2010).-. Eds. You-Jin et al., Lanzhou, China: 2010, pp 430-432.

24. R.F. Álvarez-Estrada; A. Muñoz Sudupe; L. García Gonzalo. Física Cuántica(I) (Ed. Carlos Sánchez del Río) EUDEMA, 1991, pp 242-247 (in Spanish) 